\title{
Real Analytic Maps on Manifolds
}

\section{P. T. CHURCH ${ }^{1} \&$ W. D. NATHAN ${ }^{2}$}

\author{
Communicated by Andrew Wallace
}

1. Introduction. This paper is concerned with the structure, both analytic and topological, local and global, of real analytic $\left(C^{\omega}\right)$ maps $f: M^{n} \rightarrow N^{P}$ on manifolds, especially insofar as this structure differs from that of $C^{\infty}$ maps.

1.1. Definition. For any map $f: M^{n} \rightarrow N^{n}$ the branch set $B_{f}$ is the set of $y \varepsilon M^{n}$ such that $f$ fails to be a local homeomorphism at $y$. More generally, for $f: M^{n} \rightarrow N^{P}, y \notin B_{f}$ if and only if $f$ at $y$ is locally $C^{0}$ (topologically) equivalent (2.2) to the projection map $\pi: R^{n} \rightarrow R^{P}$ defined by $\pi_{i}(x)=x_{i}$ for $i \leqq$ $\min (n, p)$ and $\pi_{i}(x)=0$ otherwise.

We prove that for a $C^{\omega} f$ with $n=p$ there is an "analytic" triangulation of $M^{n}$ in which $B_{f}$ is a subpolyhedron (5.2). The $C^{\infty}$ analog is false.

If $f: M^{n} \rightarrow N^{n}$ is complex analytic $\left(C^{\Omega}\right)$, then $B_{f}$ is exactly the set $R_{n-1}(f)$ of zeros of the Jacobian determinant of $f[2 ;$ p. 179]. If $f$ is real analytic, then $B_{f} \subset R_{n-1}(f)$ (the Inverse Function Theorem), but they need not be equal (let $M^{n}=N^{n}=R$ and $f(x)=x^{3}$ ). A natural question arises: Is $B_{f}$ some (other) analytic set (variety)? Due to the vagaries of real (as compared with complex) analytic sets [21; p. 106-109], we have been unable to decide in general. For $n \leqq 2$ it is true, and in general there is an analytic set $V$ with $B_{f} \subset V$ and $\operatorname{dim}$ $B_{f}=\operatorname{dim} V$, and, if $\operatorname{dim} B_{f} \neq n-2$, there is also an analytic set $E$ with $V-$ $E \subset B_{f}$ and $\operatorname{dim} E<\operatorname{dim} B_{f}$ (5.4). The cyclicness of $B_{f}$ is also discussed.

It is shown (4.4) that a $C^{\omega}$ map $f: M^{n} \rightarrow N^{P}$ with $\operatorname{dim} f^{-1}(y) \leqq n-p$ for each $y \varepsilon N^{P}$ has a local canonical analytic structure except on a set of dimension at most $n-2$, and, as a corollary, that a $C^{\omega}$ open map has $\operatorname{dim} B_{f} \leqq n-2$ (4.6). In (6.2) it is observed that a proper (2.3) $C^{\omega}$ map with $n=p$ and dim $B_{f}<n / 2$ is necessarily a local homeomorphism. The $C^{\omega}$ versions of all these statements are false, and the proper hypothesis is essential in the last statement. Related open questions are raised in (6.4).

2. Conventions and definitions. The theory of analytic sets is used extensively in this paper, and for the reader who is unfamiliar with it we recommend

Supported in part by an N.S.F. Senior Postdoctoral Fellowship at the Institute for Advanced Study $^{1}$, N.S.F. Grant GP-6871',2, and a N.A.S.A. Fellowship ${ }^{2}$. 
[21] for background. We shall use its notation except for germs (2.1) and its terminology except that regular points of an analytic set [21; p. 36, Definition 2] are called simple points here to avoid some possible confusion. In particular, an analytic set is closed [21; pp. 5-6, Definition 1]. Information about analytic sets sufficient for our purposes is contained in the following parts of [21]: III, Sect. 1, pp. 31-42; IV, Sect. 1, pp. 64-73; V, Sect. 1, pp. 91-96; and pp. 104-109. Other valuable references are [15] and [16].

2.1. Notation. The fields of real and complex numbers are denoted by $R$ and $C$, respectively, and either one is denoted by $F$. Coordinates are written down $x_{i},\left(x_{1}, \cdots, \hat{x}_{p}, \cdots, x_{n}\right)$ indicates that the $p$ th coordinate is omitted, and the $n$-sphere $S^{n}$ is $\left\{x \varepsilon R^{n+1}: \sum_{i}\left(x_{i}\right)^{2}=1\right\}$. For $k<n, F^{k}$ is identified with $\left\{x \varepsilon F^{n}: x_{i}=0\right.$ for $\left.i>k\right\}$, and, in particular, $0 \varepsilon F^{n}$ for all $n$.

A map $f: X \rightarrow Y$ is a continuous function, and, for $A \subset X, f \mid A$ is the restriction of $f$ to $A$. The composition of the functions $f$ followed by $g$ is denoted by $g \circ f$, and the germ of a function $f$ or a set $A$ at $x$ (defined in [15; pp. 65 and 86]) is denoted by ${ }_{x} f$ or ${ }_{x} A$, respectively. For $f^{*}$ and $A^{*}$ see (2.4), (2.5), (2.6) and (2.7), and for the maps $F_{n, d}$ and $g^{k}$ see (3.4). If $f: X \rightarrow F^{P}, f_{i}$ is the $i$ th coordinate function.

A symbol such as $M^{n}$ refers to a separable $n$-manifold without boundary, and the statement that $f: M^{n} \rightarrow N^{P}$ is (differentiable of class) $C^{m}$ implies that the manifolds are $C^{m}$. A $C^{0}$ manifold, map, or diffeomorphism is a topological manifold, map, or homeomorphism, respectively. A $C^{\omega}$ (resp., $C^{\Omega}$ ) manifold or map is a real (resp., complex) analytic manifold or map, and in the latter case its dimension is complex dimension. The derived map (Jacobian matrix) of $f$ is denoted by $D f$. For $R_{q}(f)$, see (2.8).

Homology and cohomology groups are Cech, or, equivalently [27; p. 334, Cor. 8], Alexander-Spanier, and they agree with the simplicial groups on finite polyhedra [11; p. 100], e.g. (5.1) compact analytic sets; $H_{c}^{q}$ refers to the $q$ th cohomology group with compact supports, and $Z_{2}$ is the field of order 2 . The closure of a subset $A$ is denoted by $\bar{A}$ or $C l[A]$.

2.2. Definitions. Two $C^{m}(m=0,1, \cdots ; \infty ; \omega ;$ or $\Omega) \operatorname{maps} f: M^{n} \rightarrow N^{P}$ and $g: K^{n} \rightarrow L^{P}$ are $C^{m}$ equivalent if and only if there exist $C^{m}$ diffeomorphisms $\alpha: M^{n} \rightarrow K^{n}$ and $\beta: L^{P} \rightarrow N^{P}$ such that $f=\beta \circ g \circ \alpha$. For $x \varepsilon M^{n}$ and $y \varepsilon K^{n}$ the germs ${ }_{x} f$ and ${ }_{y} g$ are $C^{m}$ equivalent if and only if there exist $C^{m}$ germs of diffeomorphisms ${ }_{x} \alpha$ and ${ }_{g(y)} \beta$ such that $\alpha(x)=y, \beta(g(y))=f(x)$, and ${ }_{g(y)} \beta \circ{ }_{y} g \circ{ }_{x} \alpha={ }_{x} f$. In this case we say that $f$ at $x$ and $g$ at $y$ are locally $C^{m}$ equivalent. ( $C^{0}$ equivalent maps are sometimes called topologically equivalent.)

2.3. Definitions. A map $f: X \rightarrow Y$ is proper if $f^{-1}(K)$ is compact whenever $K$ is compact; it is light (resp., monotone; acyclic mod 2) if, for each $y \varepsilon Y$, dim $f^{-1}(y) \leqq 0$ (resp., $f^{-1}(y)$ is connected; $f^{-1}(y)$ has the mod 2 homology (or, equivalently $[17 ;$ p. 137, $(G)]$, cohomology) groups of a point). 
It is natural to consider monotone and light maps because any proper map $f: X \rightarrow Y$ on locally compact metric spaces can be factored uniquely as $h \circ g$, where $g: X \rightarrow W$ is monotone and $h: W \rightarrow Y$ is light [34; p. 141]. A complex analytic variant of this theorem is given in [25; p. 165, Satz 4].

2.4. Definition. Let $M^{n}$ be $C^{\omega}$. A complexification [33; p. 133] of $M^{n}$ is a pair $\left(M^{*}, \phi\right)$, where $M^{*}$ is a $C^{\Omega} n$-manifold and $\phi: M^{n} \rightarrow M^{*}$ is a $C^{\omega}$ embedding, such that, for every $x \varepsilon M^{*}$, there are an open neighborhood $U^{*}$ of $x$ in $M^{*}$, an open subset $V \subset C^{n}$, and a $C^{\Omega}$ diffeomorphism $\psi: U^{*} \rightarrow V$ with $\psi\left(\phi\left(M^{n}\right) \cap U^{*}\right)=$ $R^{n} \cap V$.

Complexifications exist; moreover, if $\left(M_{1}^{*}, \phi_{1}\right)$ and $\left(M_{2}^{*}, \phi_{2}\right)$ are two complexifications of $M^{n}$, there is a $C^{\Omega}$ diffeomorphism of an open neighborhood of $\phi_{1}\left(M^{n}\right)$ in $M_{1}^{*}$ onto an open neighborhood of $\phi_{2}\left(M^{n}\right)$ in $M_{2}^{*}$ extending the $C^{\omega}$ diffeomorphism $\phi_{2} \circ \phi_{1}^{-1}$. As a result, a unique germ of $M^{*}$ at $M^{n}$ is defined, and we may suppose that $M^{n} \subset M^{*}$, i.e., $\phi$ is inclusion.

2.5. Definition. Given complexifications $\left(M^{*}, \phi\right)$ and $\left(N^{*}, \theta\right)$ of $C^{\omega}$ manifolds $M^{n}$ and $N^{P}$, respectively, and given a $C^{\omega}$ map $f: M^{n} \rightarrow N^{P}$, a complexification $f^{*}: M^{*} \rightarrow N^{*}$ is a $C^{\Omega}$ map such that $f^{*} \circ \phi=\theta \circ f$. Complexifications exist and any two agree in some neighborhood of $\phi\left(M^{n}\right)$ [26; p. 190], i.e., the germ of $f^{*}$ at $\phi\left(M^{n}\right)$ is unique. For convenience we again suppose that $\phi$ and $\theta$ are inclusion maps, so that $f=f^{*} \mid M$.

2.6. Definition. A real analytic subset $W \subset M^{n}$ of a $C^{\omega}$ manifold is called $C$-analytic if and only if, given any complexification $\left(M^{*}, \phi\right)$ of $M^{n}$ (take $\phi$ to be inclusion), there is an open neighborhood $U^{*}$ of $W$ in $M^{*}$ and a complex analytic subset $W^{\prime} \subset U^{*}$ such that (ß) $W^{\prime} \cap M^{n}=W$ [33; top of p. 153]. The intersection $\hat{W}$ of all germs at (the $C$-analytic set) $W$ of such complex analytic sets is the (unique) minimal germ at $W$ of a complex analytic subset (of a complexification of $M^{n}$ ) satisfying (P) [33; top of p. 154]. A complex analytic set $W^{*}$ representing $\hat{W}$ in some complexification $M^{*}$ of $M^{n}$ is called a complexification of $W$.

Not every real analytic set is $C$-analytic $[21 ;$ p. 104 , Example 2 and p. 104, Proposition 15], but locally each is. Thus we can define the complexification of the germ ${ }_{y} W$ of a real analytic set [21; p. 92].

$C$-irreducible $C$-analytic sets and components are defined in [33; p. 155].

With some abuse of notation we will refer to the complexification of $f, M^{n}$, or $W$, and equalities and inclusions of complexifications. In many cases the corresponding statement about germs is really meant.

2.7. Remark. Primarily we are concerned with real analytic maps in this paper. Many of the remarks, lemmas, and even one theorem are given simultaneously in real and complex versions, however, and the complex proofs are substantially the same as, or are simplifications of, the real proofs. Thus, the complex proofs are omitted in general. In one case we need a complex analytic lemma to prove a real analytic theorem, viz. (3.9) for (5.4). 
For convenience in proving real and complex analytic versions of a result simultaneously, we agree that for complex analytic manifolds $M$, functions $f$, sets $W$, and set germs ${ }_{y} W$, we have $M^{*}=M, f^{*}=f, W^{*}=W$, and $\left({ }_{y} W\right)^{*}={ }_{y} W$. Also, in the complex case $C$-analytic and $C$-irreducible mean merely analytic and irreducible.

2.8. Definition. For $C^{1}$ maps $f: M^{n} \rightarrow N^{P}$ the set of points in $M^{n}$ at which the rank of $f$ is at most $q(q=0,1, \cdots)$ is denoted by $R_{q}(f)$.

2.9. Remark. If $f: M^{n} \rightarrow N^{P}$ is $C^{\omega}$, then $R_{q}(f)(q=0,1, \cdots)$ and $f^{-1}(y)$ $\left(y \varepsilon N^{n}\right)$ are $C$-analytic. Any $C^{\omega}$ submanifold $K^{\alpha}$ of $M^{n}$ is $C$-analytic, and if connected is $C$-irreducible.

Proof. Observe that $R_{q}(f)=M^{n} \cap R_{a}\left(f^{*}\right)$ and $f^{-1}(y)=M^{n} \cap\left(f^{*}\right)^{-1}(y)$. Since coherence [21; p. 93, Definition 2] of an analytic set is a local property and $R^{a}$ is a coherent subset of $R^{n}, K^{a}$ is a coherent analytic subset of $M^{n}$. A fortiori $K^{\alpha}$ is $C$-analytic [33; p. 153, Proposition 10b)]. If $K^{\alpha}$ is connected, then $K^{*}$ is connected, so that ([21; p. 67 , Theorem 1$]$ and [33; p. 155, Proposition 11]) $K^{\alpha}$ is $C$-irreducible.

\section{Technical lemmas.}

3.1. Lemma. Let $f: M^{n} \rightarrow N^{P}$ be $C^{m}(m=\omega$ or $\Omega)$, and let $W \subset M^{n}$ be a $C$-irreducible $C$-analytic subset of dimension $\rho$. Let $r \leqq \rho$ be the maximal integer such that there is a simple point $x$ of $W$ with $\operatorname{dim}{ }_{x} W=\rho$ and the restriction ${ }_{x}(f \mid W)$ has rank $r$ at $X$. Then there is a $C$-analytic set $E \subset W$ such that $\operatorname{dim} E<\rho$, each point $y$ of $W-E$ is simple with dimension $\rho$, and the restriction ${ }_{\nu}(f \mid W)$ has rank $r$.

Proof. See (2.7). For a sufficiently small neighborhood $U^{*}$ of $M$ in $M^{*}$, the complexification $W^{*}$ in $U^{*}$ is irreducible [33; p. 155, Proposition 11], and so has constant dimension $\rho[21 ;$ p. 69 , Corollary 3$]$.

Define $X^{*}$ as the union of the singular set of $W^{*}$, together with the set of simple points $y$ of $W^{*}$ at which the restriction map ${ }_{y}\left(f^{*} \mid W^{*}\right)$ has rank less than $r$. Then $X^{*} \subset W^{*}$, while from an hypothesis $X^{*} \neq W^{*}$; we now prove that $X^{*}$ is a complex analytic subset of $W^{*}$, from which it follows that dim $X^{*}<\rho[21 ;$ p. 69 , Corollary 4].

Choose $z \varepsilon W^{*}$. By [32; p. 531, (15.1)], there is an open neighborhood $V$ of $z$ (in $U^{*}$ ) and a set of complex analytic vector fields $v^{(k)}(k=1,2, \cdots, h)$ defined on $V$ such that each $v^{(k)}(q)=0$ for each $q$ in the singular set of $W^{*}$, and, for $q$ a simple point of $W^{*}$, each $v^{(k)}(q)$ is in the tangent space of ${ }_{a}\left(W^{*}\right)$, and they span that space. We may as well suppose that $V \subset C^{n}$ and $f^{*}: V \rightarrow C^{P}$. If $\left(D f^{*}\right)_{q}$ is the Jacobian matrix at $q$, let $A(q)$ be the $h \times p$ matrix whose $k$-th row is the vector $\left(D f^{*}\right)_{a}\left(v^{(k)}(q)\right)$, and let $g_{i}(i=1,2, \cdots, m)$ be all the $r \times r$ sub-determinant functions of $A$. Then a point $q$ of $W^{*} \cap V$ is in $X^{*}$ if and only if $g_{i}(q)=0$ for every $i$. Thus $X^{*} \cap V$ is a complex analytic subset of $W^{*} \cap V$, so that $X^{*}$ is a complex analytic subset of $W^{*}$. 
Let $E=X^{*} \cap M$. Then $\operatorname{dim} E<\rho[33 ;$ p. 155, Proposition 12], and it clearly satisfies the other properties.

3.2. Lemma. For any subset $B$ of $a C^{m}(m=\omega$ or $\Omega)$ manifold $M^{n}$, there is a smallest $C$-analytic subset $W$ of $M$ containing $B$.

Proof. Let $\mathfrak{W}$ be the set of all $C$-analytic subsets of $M$ containing $B$. Since $M \varepsilon \mathfrak{W}, \mathfrak{W} \neq \varnothing$; let $W$ be the intersection of all elements of $\mathfrak{W}$. Then $W \varepsilon \mathfrak{W}$ [33; p. 154], and $W$ is contained in every element of $\mathfrak{W}$.

3.3. Lemma. Given a $C^{m}(m=\omega$ or $\Omega)$ map $f: M^{n} \rightarrow N^{P}$ and $B \subset M^{n}$, there is a sequence of $C$-analytic subsets $W_{i}$ and $E_{i}$ of $M^{n}(i=1,2, \cdots$; all but a finite number of the sets are empty) such that:

(1) each $W_{i}$ is the union of its simple points of dimension $\operatorname{dim} W_{i}$, together with $E_{i}$, where $\operatorname{dim} E_{i}<\operatorname{dim} W_{i}$;

(2) for each $C$-irreducible component $W_{i, \nu}$ of $W_{i}$, the restriction map $f \mid\left(W_{i, \nu}-\right.$ $\left.E_{i}\right)$ has constant rank; and

(3) each $W_{i}$ is the smallest $C$-analytic set with $W_{i} \subset E_{i-1}$ and $E_{i-1} \cap B \subset$ $W_{i}\left(E_{0}=M^{n}\right)$.

Proof. See (2.7). Let $W_{1}$ be the smallest $C$-analytic subset of $M$ with $B C$ $W_{1}$ (3.2), and let $d(B)=\operatorname{dim} W_{1}$. We use induction on $d(B)$; the theorem is trivial if $d(B)=0$. Suppose that it is true for all sets $B$ with $d(B) \leqq k$, and consider a set $B$ with $d(B)=k+1$.

Let $W_{1, \nu}(\nu=1,2, \cdots)$ be the $C$-irreducible components of $W_{1}$, let $E_{1, \nu}$ be the $C$-analytic subsets of $W_{1, v}$ given by (3.1), and let $E_{1}$ be the union of the $E_{1, \nu}$ together with those $W_{1, \nu}$ with $\operatorname{dim} W_{1, \nu}<\operatorname{dim} W_{1}$ [33; p. 154]. Then $W_{1}$ and $E_{1}$ satisfy (1), (2), and (3). Let $W_{2}$ be the $C$-analytic set satisfying (3) for $i=2$ (3.2). Since $d\left(E_{1} \cap B\right) \leqq k$, the desired conclusion follows from the inductive hypothesis.

3.4. Definitions. Let $F_{n, d}: R^{n} \rightarrow R^{n}(n \geqq 2, d \geqq 2)$ be defined by $F_{n, d}\left(x_{1}, x_{2}, \cdots, x_{n}\right)=\left(u_{1}, u_{2}, \cdots, u_{n}\right)$, where $u_{j}=x_{i}$ for $j>2, u_{1}+i u_{2}=$ $\left(x_{1}+i x_{2}\right)^{d}$, and $i=\sqrt{-1}$. The branch set $B_{F}$ is an $(n-2)$-plane.

Define $g^{k}: F^{n} \rightarrow F^{P}$ by $g^{k}\left(x_{1}, x_{2}, \cdots, x_{n}\right)=\left(x_{1}, \cdots, x_{k}, 0, \cdots, 0\right)(k=0$, $1, \cdots, \min (n, p))$.

3.5. Theorem (Сhurch \& Hemmingsen). Let $f: M^{n} \rightarrow N^{n}$ be light, let $n \geqq 2$, let $\Gamma \subset N^{n}$ be a tame $k$-cell, $k \leqq n-2$, and let $B_{f} \neq \varnothing$ with $f\left(B_{f}\right) \subset \Gamma$. Then $k=n-2$, and at each point $x \in B_{f},{ }_{x} f$ is $C^{0}$ equivalent to ${ }_{0} F_{n, d}(d=2,3, \cdots)$.

See $[9 ;$ p. 608, (1.2) and (1.3)].

3.6. Remark. If $f: M^{n} \rightarrow M^{P}$ is $C^{m}(m=1,2, \cdots ; \infty)$, then there is a closed subset $A \subset M^{n}$ such that $\operatorname{dim} A \leqq n-1$, and at each point $y$ of $M^{n}-A, f$ is locally $C^{m}$ equivalent to some $g^{k}(k=0,1, \cdots, \min (n, p) ; k$ depending on $y)$.

Proof. Let $U \subset M^{n}$ be any open set, and let $y \varepsilon U$ be a point at which $f \mid U$ 
has maximal rank $k(y)(k(y)=0,1, \cdots, \min (n, p))$. By the Rank Theorem $\left[15 ;\right.$ p. 273] there is a neighborhood of $y$ on which $f$ is $C^{m}$ equivalent to $g^{k(y)}$. Thus the set $A$ of points at which $f$ is not $C^{m}$ equivalent to some $g^{k}$ is closed, and since it contains no open set, $\operatorname{dim} A \leqq n-1$ [17; p. 44, Theorem IV.3].

The situation in the $C^{\omega}$ case is nicer.

3.7. Remark. Let $M^{n}$ be connected, and let $f: M^{n} \rightarrow N^{P}$ be $C^{m}(m=\omega$ or $\Omega)$. Then there is a unique integer $k(0 \leqq k \leqq \min (n, p))$ such that the $C$-analytic set $R_{k-1}(f)$ has dimension at most $n-1$, and at every point $z$ of $M^{n}-R_{k-1}(f),{ }_{2} f$ is $C^{m}$ equivalent to ${ }_{z} g^{k}$. Thus, either $B_{f}=M^{n}$ or $\operatorname{dim} R_{\min (n, p)}(f) \leqq n-1$.

Proof. Let $k$ be the maximal value of the rank of $f$ at a point of $M^{n}$; the Rank Theorem [10; p. 273] yields the result.

3.8. Lemma. Let of : $F^{n} \rightarrow F^{P}$ be a $C^{m}$ germ $(m=1,2, \cdots ; \omega$ or $\Omega)$ with $f(0)=0$, and let ${ }_{0} \Gamma$ be a $C^{m}$ germ of a q-submanifold of $F^{n}$ such that $0 \varepsilon \Gamma$ and ${ }_{0}(f \mid \Gamma)$ has constant rank $s$. Then there exist $C^{m}$ germs at 0 of diffeomorphisms ${ }_{0} \alpha:{ }_{0} F^{n} \rightarrow{ }_{0} F^{n}$ and ${ }_{0} \beta:{ }_{0} F^{P} \rightarrow{ }_{0} F^{P}$ and $a C^{m}$ germ ${ }_{0} u:{ }_{0} F^{n} \rightarrow{ }_{0} F^{n}$ such that:

(a) ${ }_{0} u={ }_{0} \beta \circ{ }_{0} f \circ{ }_{0} \alpha$;

(b) ${ }_{0} \alpha(0)={ }_{0} \beta(0)={ }_{0} u(0)=0$;

(c) ${ }_{0} \alpha\left({ }_{0} F^{q}\right)={ }_{0} \Gamma$ and ${ }_{0} \beta\left({ }_{0} f\left({ }_{0} \Gamma\right)\right)={ }_{0} F^{s}$, so that ${ }_{0} u\left({ }_{0} F^{a}\right)={ }_{0} F^{s}$; and

(d) ${ }_{0} u_{i}(x)=x_{i}(i=1,2, \cdots, s)$.

For simplicity in the following proof, the term "map" means "germ at 0 of a map", and equality of maps or topological subspaces of $F^{n}$ means equality of germs at 0 of maps or subspaces.

Proof. There is a $C^{m}$ diffeomorphism $\gamma: F^{n} \rightarrow F^{n}$ such that $\gamma(0)=0$ and $\gamma\left(F^{a}\right)=\Gamma$. From the Rank Theorem [10; p. 273] applied to $f \circ \gamma \mid F^{\alpha}$, there are $C^{m}$ diffeomorphisms $\beta: F^{P} \rightarrow F^{P}$ and $\delta: F^{q} \rightarrow F^{q}$ such that $\beta(0)=\delta(0)=0$ and $\beta \circ f \circ \gamma \circ \delta\left(x_{1}, \cdots, x_{q}\right)=\left(x_{1}, \cdots, x_{s}, 0, \cdots, 0\right)$. Define $\sigma: F^{n} \rightarrow F^{n}$ by $\sigma\left(x_{1}, \cdots, x_{n}\right)=\left(\delta_{1}(x), \cdots, \delta_{q}(x), x_{q+1}, \cdots, x_{n}\right)$, and let $\beta \circ f \circ \gamma \circ \sigma$ be denoted by $h$. Then $h\left({ }_{0} F^{a}\right) \subset F^{s}$. For $i=j \leqq s, D_{i} h_{j}(0)=1$; for $s+1 \leqq i=$ $j \leqq q, D_{i} h_{j}(0)=0$; and for $i \neq j, i \leqq q, j \leqq p, D_{i} h_{j}(0)=0$ also. From [6; p. $87,(1.1)$; its proof applies in the analytic cases] there are $C^{m}$ diffeomorphisms $\zeta: F^{n} \rightarrow F^{n}$ and $\eta: F^{P} \rightarrow F^{P}$ such that $g=\eta \circ h \circ \zeta$ has $g_{i}(t)=$ $t_{i}(i=1,2, \cdots, s)$. Moreover we may suppose that $\eta$ is the identity map [8; p. 415, (2.3)].

Let $\Psi_{v, w}$ be the subspace of $F^{v}$ defined by $t_{i}=0(i=1,2, \cdots, w)$, and for a $C^{m}$ submanifold $K \subset F^{v}$ let $T_{y} K$ be the tangent space of $K$ at $y$. Since $\zeta$ is a diffeomorphism, $\operatorname{dim}\left(D \zeta_{0}\left(T_{0} \Psi_{n, s}\right)\right)=n-s ;$ since $g\left(\Psi_{n, s}\right) \subset \Psi_{p, s}$,

$$
D h_{0}\left(D \zeta_{0}\left(T_{0} \Psi_{n, s}\right)\right)=D g_{0}\left(T_{0} \Psi_{n, s}\right) \subset T_{0} \Psi_{p, s} \text {. }
$$

Now $D h_{0}\left(\partial / \partial x_{k}\right)=\partial / \partial u_{k}+\sum_{r>s} D_{k} h_{r}(0) \cdot \partial / \partial u_{r}(k=1,2, \cdots, s)$, so that $\operatorname{dim} D h_{0}\left(F^{s}\right)=s$ and $D h_{0}\left(F^{s}\right) \cap T_{0} \Psi_{p, s}=\{0\}$. Thus $F^{s} \cap D \zeta_{0}\left(T_{0} \Psi_{n, s}\right)=\{0\}$, so that the vectors $\partial / \partial x_{k}(k=1,2, \cdots, s)$ and $D \zeta_{0}\left(\partial / \partial t_{i}\right)=\sum_{r} D_{i} \zeta_{r}(0) \cdot \partial / \partial x_{r}$ 
$(i=s+1, s+2, \cdots, n)$ constitute a basis for $T_{0} F^{n}$. The components of these vectors define an $(n \times n)$-nonsingular matrix $A_{i, j}$ with $A_{i, j}(i, j=1,2, \cdots, s)$ the identity $(s \times s)$-matrix. Thus the matrix $D_{i} \zeta_{j}(0)(i, j=s+1, s+2, \cdots, n)$ is nonsingular, so that $D_{i} \zeta_{i}(0)(i=s+1, s+2, \cdots, n ; j=q+1, q+2, \cdots, n)$ has rank $n-q$. (In effect the notion of transverse regularity [31; p. 22] has been used in the above argument.)

We may as well suppose that $D_{i} \zeta_{j}(0)(i, j=q+1, q+2, \cdots, n)$ is nonsingular. Let $\omega: F^{n} \rightarrow \Psi_{n, q}$ be defined by $\omega_{i}=\zeta_{i}$. By the Implicit Function Theorem [10; p. 265], there is a unique $C^{m}$ function $\lambda: F^{\alpha} \rightarrow F^{n-q}$ such that $\lambda(0)=0$ and (the germ at 0 of) the graph of $\lambda$ is the set of zeros of $\omega$, i.e., $\zeta_{i}\left(t_{1}, \cdots, t_{q}, \lambda_{1}\left(t_{1}, \cdots, t_{q}\right), \cdots, \lambda_{n-q}\left(t_{1}, \cdots, t_{q}\right)\right)=0(i=q+1$, $q+2, \cdots, n)$. Define $\xi: F^{n} \rightarrow F^{n}$ by $\xi_{i}(t)=t_{i}(i=1,2, \cdots, q)$ and $\xi_{i}(t)=$ $t_{i}+\lambda_{i-q}\left(t_{1}, \cdots, t_{q}\right)(i=q+1, q+2, \cdots, n)$. Since $D \xi(0)$ is non-singular, $\xi$ is a diffeomorphism. Let $\alpha=\gamma \circ \sigma \circ \zeta \circ \xi$; then $\alpha\left(F^{a}\right)=\Gamma$, and $\beta \circ f \circ \alpha$ is the desired map $u$.

3.9. Proposition. Let $f: M^{n} \rightarrow N^{P}$ be $C^{m}(m=\omega$ or $\Omega)$, let $\Gamma$ be a $C^{m}$ connected $(n-1)$-submanifold of $M^{n}$ such that $f \mid \Gamma$ has rank $p-1$ at every point, and let $R_{p-1}(f) \subset \Gamma$.

(i) Then there is a unique natural number $k$ and an analytic subset $A \subset \Gamma$ with $\operatorname{dim} A \leqq n-2$ such that for each $y \varepsilon \Gamma-A$ the germ ${ }_{y} f$ is $C^{m}$ equivalent to ${ }_{0} h$, where $h: F^{n} \rightarrow F^{P}$ is defined by $h_{i}(x)=x_{i}(i<p)$ and $h_{p}(x)=\left(x_{p}\right)^{k}$.

(ii) In case $n=p$ and $R_{p-1}\left(f^{*}\right) \subset \Gamma^{*}, A=\varnothing$.

To see that $A$ is needed, or, in case $n=p$, that $R_{p-1}\left(f^{*}\right)$ must be contained in $\Gamma^{*}$ if $A$ is to be empty, consider the maps $f: F^{2} \rightarrow F^{2}$ and $f_{2}$ defined by $f_{1}(x)=x_{1}$ and $f_{2}(x)=\left(x_{1}\right)^{2} \cdot\left(x_{2}\right)^{2}+\left(x_{2}\right)^{4}$ (see the end of the following proof).

Proof. From (3.7) and the hypothesis $R_{p-1}(f) \subset \Gamma, \operatorname{dim} R_{p-1}\left(f^{*}\right)<n$. Let $S$ be $R_{p-1}\left(f^{*}\right)$ if $\operatorname{dim} R_{p-1}\left(f^{*}\right)<n-1$ and the singular set of $R_{p-1}\left(f^{*}\right)$ if $\operatorname{dim} R_{p-1}\left(f^{*}\right)=n-1$. Since $S \cap M^{n}$ is a $C$-analytic subset of $M^{n}$ with dimension at most $n-2([21 ;$ p. 66 , Proposition 2$],(2.6)$, and [33; p. 155, Proposition $12]), \operatorname{dim}\left(S \cap R_{p-1}(f)\right) \leqq n-2$. Except in the proof of (ii), we may include the $C$-analytic set ([33; p. 154] and (2.8)) $S \cap R_{p-1}(f)$ in $A$, so we may as well suppose (in both cases) that $R_{p-1}\left(f^{*}\right) \subset \Gamma^{*}$.

The hypothesis on the rank of $f \mid \Gamma$ implies that $n \geqq p$. Let $h^{k}$ be the map defined above, and let $y \varepsilon \Gamma$. There exist $C^{m}$ germs at 0 of diffeomorphisms ${ }_{0} \sigma: F^{n} \rightarrow M^{n}$ and ${ }_{0} \tau: F^{P} \rightarrow N^{P}$ such that ${ }_{0} \sigma(0)=y$ and ${ }_{0} \tau(0)=f(y)$. Let ${ }_{0} u$ be the germ given by (3.8) for ${ }_{f(y)} \tau^{-1} \circ{ }_{\nu} f{ }_{0} \sigma$; let $\rho: F^{n} \rightarrow F^{n}$ be defined by $\rho_{i}(x)=x_{i}$ if $i \neq p, n, \rho_{p}(x)=x_{n}$, and $\rho_{n}(x)=x_{p}$; let ${ }_{0} v={ }_{0} u \circ{ }_{0} \rho$; and let $\Delta \subset F^{n}$ be the subspace defined by $x_{p}=0$. Then (1) ${ }_{0} v(0)=0,(2)_{0} v$ is $C^{m}$ equivalent to ${ }_{y} f$, with ${ }_{0} \Delta$ corresponding to ${ }_{y} \Gamma,(3) R_{p-1}\left({ }_{0} v^{*}\right) \subset{ }_{0} \Delta^{*},(4){ }_{0} v\left({ }_{0} \Delta\right) \subset$ ${ }_{0} F^{p-1}$, and $(5){ }_{0} v_{i}(x)=x_{i}(i=1,2, \cdots, p-1)$.

Let $\Lambda=\left\{x \varepsilon F^{n}: x_{i}=0\right.$ for $\left.i \leqq p-1\right\}$, and for $c \varepsilon F^{n}$ let $L(c) \subset \Lambda$ be the line defined by $x_{i}=c_{j} x_{p}(j=p+1, p+2, \cdots, n)$. If ${ }_{0} v_{p}\left({ }_{0} \Lambda\right)=\{0\}$, it would 
follow that ${ }_{0} \Lambda \subset{ }_{0} R_{p-1}(v)$, contradicting (3). Thus, for some $c,{ }_{0} v\left(_{p}(L(c))\right) \neq\{0\}$. The linear isomorphism $\psi: F^{n} \rightarrow F^{p}$ defined by $\psi_{i}(x)=x_{i}(j \leqq p)$ and $\psi_{i}(x)=$ $x_{i}+c_{j} x_{p}(j>p)$ maps $L(0)$ onto $L(c)$; we may replace ${ }_{0} v$ by ${ }_{0} v \circ{ }_{0} \psi^{-1}$, so that we may as well suppose that ${ }_{0} v(L(0)) \neq\{0\}$, i.e., (6) ${ }_{0} v\left(0, \cdots, 0, x_{p}\right.$, $0, \cdots, 0) \not \equiv 0$.

There is a connected open neighborhood $V$ of 0 in $F^{n}$ and a $C^{m}$ function $v: V \rightarrow F^{v}$ representing ${ }_{0} v$ such that (1) for an open neighborhood $Y^{v}$ of $f(y)$ in $N^{p}$ there exist diffeomorphisms $\theta^{v}: V \rightarrow \theta^{v}(V) \subset M^{n}$ and $\phi^{v}: Y^{v} \rightarrow$ $\phi^{v}\left(Y^{v}\right) \subset F^{p}$ with $v=\phi^{v} \circ f \circ \theta^{v} C^{m}$ equivalent to $f \mid \theta^{v}(V): \theta^{v}(V) \rightarrow Y^{v} ;(2)$ $\theta^{*}(0)=y$ and $\phi^{v}(f(y))=0$; (3) $R_{p-1}\left(v^{*}\right) \subset \Delta^{*} ;(4) \theta^{v}(V) \cap \Gamma=\theta^{v}(V \cap \Delta)$ is connected, and $v(V \cap \Delta) \subset F^{p-1} ;(5)$ the power series expansion

$$
v_{p}(x)=\sum_{j \geq 0} a_{v, j}\left(x_{1}, \cdots, \hat{x}_{p}, \cdots, x_{n}\right) \cdot\left(x_{p}\right)^{j}
$$

and $v_{i}(x)=x_{i}(i<p)$ define $v$ throughout $V$; and (6) for each $z \varepsilon V \cap \Delta$ $v_{p}\left(z_{1}, \cdots, z_{p-1}, x_{p}, z_{p+1}, \cdots, z_{n}\right) \neq \equiv$ (as a function of $x_{p}$ alone).

The $C^{m}$ functions $a_{v, i}$ are defined on $V \cap \Delta$, and (from (4)) $a_{v, 0} \equiv 0$. Let $k(v)$ be the least $j$ such that $a_{v, j} \neq \equiv 0$.

We now observe that for any point $z \varepsilon V \cap \Delta$ with $a_{v, k(v)}\left(z_{1}, \cdots, \hat{z}_{p}, \cdots, z_{n}\right)$ $\neq 0,{ }_{z} v$ is $C^{m}$ equivalent to ${ }_{0} h^{k(v)}$ (the germ at 0 of $h^{k(v)}$ ). By translations we may as well suppose that $z=v(z)=0$. Write ${ }_{0} v_{p}(x)=\left(x_{p}\right)^{k(v)} \cdot{ }_{0} \lambda(x)$, where ${ }_{0} \lambda(0) \neq 0$. Let ${ }_{0} \mu$ be a $k(v)$ th root of ${ }_{0} \lambda$, i.e., $\left({ }_{0} \mu(x)\right)^{k(v)}={ }_{0} \lambda(x)$. The $C^{m}$ germ ${ }_{0} \nu: R^{n} \rightarrow R^{p}$ defined by ${ }_{0} \nu_{i}(x)=x_{i}(i \leqq p-1)$ and ${ }_{0} \nu_{p}(x)=x_{p} \cdot{ }_{0} \mu(x)$ has rank $p$ at 0 , so there is a $C^{m}$ diffeomorphism germ ${ }_{0} \zeta: R^{n} \rightarrow R^{n}$ such that $\left.{ }_{0} \nu \circ{ }_{0} \zeta\right)_{i}(x)=x_{i}$ $(i=1,2, \cdots, p)[19$; pp. $7-8$; the proof applies in the analytic cases]. Since ${ }_{0} \nu_{i}(x)={ }_{0} v_{i}(x)(i<p)$ and ${ }_{0}\left(\nu_{p}(x)\right)^{k(v)}={ }_{0} v_{p}(x),{ }_{0} v_{0} \zeta={ }_{0} h^{k(v)}$.

The set of $z \varepsilon V \cap \Delta$ with $a_{v, k(v)}\left(z_{1}, \cdots, \hat{z}_{p}, \cdots, z_{p}\right)=0$ is an (at most) $(n-2)$-dimensional analytic subset $A_{v}$ of $V$ [33; p. 155 , Corollaire]. We next observe that for $n=p, A_{v}=\varnothing$. Let $z \varepsilon A_{v}$; as above we may suppose that $z=v(z)=0$. There is a sequence $\left\{{ }^{i} y\right\}$ in $V \cap \Delta$ with $^{i} y \rightarrow 0$ and $a_{v, k(v)}\left({ }^{j} y\right) \neq 0$. Let ${ }^{0} y=0$, and let ${ }_{0} \xi_{j}: C \rightarrow C$ be defined by ${ }_{0} \xi_{j}(t)=D_{n}\left({ }_{0} v_{n}^{*}\right)\left({ }^{i} y_{1}, \cdots,{ }^{i} y_{n-1}, t\right)$ (where $j=0,1, \cdots$ ). Choose $\epsilon>0$ sufficiently small that for $|t| \leqq \epsilon$ each $\xi_{j}$ is defined and $\xi_{0}(t)=0$ only at $t=0$. Thus, there is an integer $J$ such that $\xi_{i}(t) \neq 0$ for $|t|=\epsilon$ and $j \geqq J$. The multiplicity of the zero of ${ }_{0} \xi_{0}$ (resp., ${ }_{0} \xi_{j}$ with $j>0$ ) is greater than (resp., equal to) $k(v)-1$. By Rouché's Theorem [10; p. 243, (9.17.3)] each $\xi_{i}$ has more than $k(v)-1$ zeros counting multiplicity for $j \geqq J$ and $|t|<\epsilon$. Thus there is a $t_{i} \neq 0$ with $\left|t_{j}\right|<\epsilon$ and $\xi_{i}\left(t_{j}\right)=0$; in fact, $t_{i} \rightarrow 0$. As a result, $D v^{*}$ is a singular linear transformation at $\left({ }^{i} y_{1}, \ldots,{ }^{i} y_{n-1}, t_{i}\right)$, contradicting (3).

Suppose that there exists a point in $\Gamma$ at which $f$ has $\operatorname{rank} p$. Let $A=R_{p-1}(f)$; then $\operatorname{dim} A \leqq n-2$ ([33; p. 155, Corollaire] and (2.9)). From the Rank Theorem [10; p. 273] it follows that, for each $y \varepsilon \Gamma-A,{ }_{y} f$ is $C^{m}$ equivalent to ${ }_{0} h^{\mathbf{1}}$, and (from rank considerations) it is not $C^{m}$ equivalent to ${ }_{0} h^{k}$ for any $k>1$. Suppose that $n=p, y \varepsilon \Gamma$, and $v$ satisfies $(1)-(6)$ at $y$; then $k(v)=1$, and we have 
just shown that $a_{v, 1}$ is never 0 , so that $A=\varnothing$ in case $n=p$. Thus we may as well suppose that $\Gamma \subset R_{p-1}(f)$, so that $\Gamma^{*}=R_{p-1}\left(f^{*}\right)$.

Let $w: W \rightarrow F^{n}$ be another $C^{m}$ function satisfying (1) - (6) (at $y$ ); by restricting $v$ and $w$ to smaller neighborhoods of 0 we may suppose that $\theta^{v}(V)=$ $\theta^{w}(W)$, and that $1<k(w) \leqq k(v)$. We will prove that $\left(^{*}\right)$ if $z \varepsilon \theta^{w}$ $\left((W \cap \Delta)-A_{w}\right)$, then there exists $j \leqq k(w)$ such that $a_{v, j}\left(\left(\theta^{v}\right)^{-1}(z)\right) \neq 0$; since we may complexify, it suffices to prove $\left({ }^{*}\right)$ in the complex $(m=\Omega)$ case, and we suppose the contrary.

We may as well suppose (by translations) that $\left(\theta^{v}\right)^{-1}(z)=\left(\theta^{w}\right)^{-1}(z)=0$ and $v(0)=w(0)=0$, so that $a_{v, j}(0, \cdots, 0)=0$ for every $j \leqq k(w)$. We may also suppose that $w=h^{k(w)}$. Let $\alpha$ and $\beta$ be the diffeomorphisms $\left(\theta^{v}\right)^{-1} \circ \theta^{w}$ and $\phi^{w} \circ\left(\phi^{v}\right)^{-1}$; then $\alpha(0)=\beta(0)=0$ and $w=\beta \circ v \circ \alpha$. Since ${ }_{0} \Delta={ }_{0} R_{p-1}(v)=$ ${ }_{0} R_{p-1}(w),{ }_{0} \alpha^{-1}\left({ }_{0} \Delta\right)={ }_{0} \Delta$, so that ${ }_{0} \alpha^{-1}(L(0))$ is transverse to ${ }_{0} \Delta$ (where $L(0)$ is defined by $\left.x_{\imath}=0(i \neq p)\right)$. We may suppose that $W$ is a polydisk [15; p. 1] and that it is small enough that $W \cap \alpha^{-1}(L(0))$ (i.e., $\alpha^{-1}(L(0))$ ) is transverse to and meets each $(n-1)$-plane $x_{p}=$ constant. Thus, for each $q \varepsilon W-\Delta$, $w^{-1}(w(q))$ consists of exactly $k(w)$ disjoint $(n-p)$-polydisks (since $w=h^{k(w)}$ ); in particular, for $q \varepsilon \alpha^{-1}(L(0))-\{0\}, w^{-1}\left(w(q) \cap \alpha^{-1}(L(0))\right.$ has exactly $k(w)$ points. On the other hand, $v_{p}\left(0, \cdots, 0, x_{p}, 0, \cdots, 0\right)=\left(x_{p}\right)^{d} \cdot \pi\left(x_{p}\right)$, where $d$ is a natural number (by (6)) greater than $k(w)$ and $\pi$ is analytic with $\pi(0) \neq 0$, so that for $r \varepsilon(L(0) \cap V)-\{0\}$ sufficiently near $0, v^{-1}(v(r)) \cap L(0)$ has precisely $d$ points. From the $C^{m}$ equivalence $w=\beta \circ v \circ \alpha$ a contradiction results, and $\left(^{*}\right)$ is proved.

Since $\operatorname{dim}\left(\theta^{v}\left(A_{v}\right) \cup \theta^{w}\left(A_{w}\right)\right) \leqq n-2$, there exists $z \varepsilon \theta^{w}(W \cap \Delta)-$ $\left(\theta^{v}\left(A_{v}\right) \cup \theta^{w}\left(A_{w}\right)\right)$; it follows from $\left(^{*}\right)$ that $k(v) \leqq k(w)$, so that $k(v)=k(w)$. Thus a unique natural number $k$ on $\Gamma$ is defined. It also follows from $\left(^{*}\right)$ that $\theta^{v}\left(A_{v}\right)=\theta^{w}\left(A_{w}\right)$; the union of these sets (for $y \varepsilon \Gamma$ ) is thus the desired analytic set of $A$.

The following result is not needed in this paper, but it is related to the previous lemmas and is thus given here.

3.10. Proposition. Let $f: M^{n} \rightarrow N^{n}$ be a $C^{1}$ map, let $\Gamma$ be a $C^{1}(n-1)$-submanifold of $M^{n}$ such that $B_{f} \subset \Gamma$ and $f \mid \Gamma$ is a $C^{1}$ embedding, and let $y \varepsilon \Gamma$. Then the germ ${ }_{y} f$ is $C^{0}$ equivalent to ${ }_{0} h$, where $h: R^{n} \rightarrow R^{n}$ is defined by $h_{i}(x)=x_{i}(i<n)$, $h_{n}(x)=\left(x_{n}\right)^{k}$, and $k=1$ or 2 .

Proof. We may suppose that $M^{n}=N^{n}=R^{n}, y=0=f(y), u$ is the map given in (3.8), $u$ is defined on all of $R^{n}$, and $0 \varepsilon B_{u}$.

If $K$ is a line given by $x_{i}$ constant $(i=1,2, \cdots, n-1)$, then $u \mid K$, viewed as a map of $K$ into $K$, has $B_{u \mid K} \subset B_{u} \cap K$. Thus $B_{u \mid K} \subset\{0\}$, so that $u(K-\{0\}) \subset$ $K-\{0\}$ and $u \mid K$ is a homeomorphism on each interval of $K-\{0\}$.

Let $Q_{1, \epsilon}$ (resp., $Q_{2, \epsilon}$ ) be the set of points $x \varepsilon R^{n}$ with $\left|x_{i}\right|<1(i<n)$ and $0<x_{n}<\epsilon$ (resp., $-\epsilon<x_{n}<0$ ), where $\epsilon>0$. Then $u \mid Q_{1, \infty}$ is one-to-one and open, and thus a homeomorphism onto its image. For each $i$ there is a $j$ with $u\left(Q_{i, \infty}\right)=Q_{i, \infty}(i, j=1,2)$, and we may suppose that $u\left(Q_{1, \infty}\right) \subset Q_{1, \infty}$. If 
$u\left(Q_{2, \infty}\right) \subset Q_{2, \infty}$, then $u \mid\left(\bar{Q}_{1, \infty} \cup \vec{Q}_{2, \infty}\right)$ is a homeomorphism with image a neighborhood of $F^{n-1}$. This contradicts the fact that $0 \varepsilon B_{u}$; thus $u\left(Q_{2, \infty}\right) \subset Q_{1, \infty}$.

Choose $\eta>0$ such that $\bar{Q}_{1, \eta} \subset u\left(\bar{Q}_{1,1}\right) \cap u\left(\bar{Q}_{2,1}\right)$, and let $C_{i}=u^{-1}\left(Q_{1, \eta}\right) \cap$ $\bar{Q}_{i, 1}$. Define $v: C_{1} \cup C_{2} \rightarrow R^{n}$ by $v_{i}(x)=x_{i}(j<n)$ and $v_{n}(x)=(-1)^{i-1} u_{n}(x)$ (for $\left.x \in \varepsilon C_{i}\right)$. Then $v$ is a homeomorphism, $u_{j}\left(v^{-1}(x)\right)=x_{j}(j<n)$ and $\left(u_{n}\left(v^{-1}(x)\right)\right)=\left|x_{n}\right|$. Define $w: R^{n} \rightarrow R^{n}$ by $w_{i}(x)=x_{i}(i<n)$ and $w_{n}(x)=$ $\left(\operatorname{sign} x_{n}\right) \cdot\left(x_{n}\right)^{2}$. Then $w$ is a homeomorphism, $w_{i}\left(u\left(v^{-1}(x)\right)\right)=x_{i}(i<n)$, and $w_{n}\left(u\left(v^{-1}(x)\right)\right)=\left(x_{n}\right)^{2}$.

3.11. Remark. It is not sufficient that $f \mid \Gamma$ be a topological embedding. Let $\alpha: R^{2} \rightarrow R^{2}$ be given by $\alpha\left(x_{1}, x_{2}\right)=\left(x_{1},\left(x_{2}\right)^{2}\right)$, let $\beta: R^{2} \rightarrow R^{2}$ be the complex analytic function given by $\beta(z)=z^{3}$, and let $f=\beta \circ \alpha$. Then $B_{f}=R^{1}$, while $f$ maps $B_{f}$ homeomorphically onto itself.

If we assume that $B_{f}=f^{-1}\left(f\left(B_{f}\right)\right)$, an argument related in part to the above proof yields the conclusion with no differentiability assumption whatever.

4. Real analytic open and related maps. In [24; p. 358, Satz 28 and 29 ; cf. p. 346, Def. 12 and Def. 13, and p. 329] Remmert proved a generalizaton of the following theorem:

4.1. Theorem (REMmert). A $C^{\Omega}$ map $f: M^{n} \rightarrow N^{p}$ is open if and only if, for every $y \varepsilon N^{p}, \operatorname{dim} f^{-1}(y)=n-p$.

4.2. Proposition. If $f: M^{n} \rightarrow N^{p}$ is $C^{\omega}$ and open, then for every $y \varepsilon N^{p}$, $\operatorname{dim}$ $f^{-1}(y) \leqq n-p$; if $p=1$, equality holds.

Proposition (4.2) is not an immediate corollary of (4.1), since the complexification $f^{*}$ of a $C^{\omega}$ open map $f$ need not be open (e.g., $f(x, y)=\left(x\left(x^{2}+y^{2}\right)\right.$, $\left.\left.y\left(x^{2}+y^{2}\right)\right)\right)$.

Proof. The $C^{\omega}$ version of $[21 ;$ p. 60 , Proposition 13$]$ with $=$ replaced by $\leqq$ holds (trivially), and with it the $C^{\omega}$ version of the third paragraph of the proof of $[21 ;$ p. 132, Proposition 4$]$ is the desired proof, except that $\operatorname{dim} X \leqq m-1$ (only) and other equalities become inequalities. For $p=1$ see $[22 ;$ p. 5, (2.5)].

The converse statement is clearly false, but it is not clear whether (for $p \geqq 2$ ) equality necessarily holds for every $y$. For example, the suspension $S(g)$ of the Hopf fibering $g: S^{3} \rightarrow S^{2}[27 ;$ p. 96, (6) with $n=1]$ is $C^{0}$ equivalent to a $C^{\infty}$ map [4; p. 189, (2.4)]; is it $C^{0}$ equivalent to a $C^{\omega}$ map?

4.3. Remark. If $f: M^{n} \rightarrow N^{p}$ is $C^{m}(m=\omega$ or $\Omega)$ and open and $V \subset N^{p}$ is an analytic set, then $\operatorname{dim} f^{-1}(V) \leqq n-p+\operatorname{dim} V$.

Proof. We may suppose that $N^{p}$ is an open subset of $F^{p}$, and that $V$ is a $q$-dimensional $C$-analytic set. For $V=F^{\alpha}$ apply (4.1) or (4.2) to $\pi \circ f$, where $\pi: F^{p} \rightarrow F^{p-a}$ is defined by $\pi_{i}(x)=x_{a+i}$; the result for $V$ a manifold follows. In general, $V$ is the union of an analytic $q$-manifold $W^{q}$ and a $C$-analytic set $E$ with $\operatorname{dim} E \leqq q-1$ [33; p. 156, Proposition 13], and since $W^{\alpha}$ is the countable union of closed subsets of $F^{p}$, the conclusion follows from induction and [17; p. 30]. 
4.4. Theorem. Let $f: M^{n} \rightarrow N^{p}$ be $C^{m}(m=\omega$ or $\Omega)$ with $p>0$ and $\operatorname{dim}$ $f^{-1}(y) \leqq n-p$ for every $y \varepsilon N^{p}$. Then there is a closed set $A_{1} \cup A_{2} \subset M^{n}$ such that $A_{1}$ is analytic in $M^{n}, A_{2}$ is analytic in $M^{n}-A_{1}\left(\right.$ if $\left.n=p, A_{2}=\varnothing\right)$, dim $\left(A_{1} \cup A_{2}\right) \leqq n-2$, and, for every $z \varepsilon M^{n}-\left(A_{1} \cup A_{2}\right)$, the germs ${ }_{z} f$ and ${ }_{0} h: F^{n} \rightarrow$ $F^{p}$ are $C^{m}$ equivalent, where $h_{i}(x)=x_{i}(i<p)$ and $h_{p}(x)=\left(x_{p}\right)^{q(z)}(q(z)=1,2, \cdots)$.

Proof. We may suppose that $M^{n}$ is connected. From the hypothesis on point inverses, $n \geqq p$. By (3.7) there is a unique natural number $k$ such that at each point $a$ of $M^{n}$, except for an analytic set of dimension at most $n-1$, af is $C^{m}$ equivalent to ${ }_{a} g^{k}(3.4)$, so that $\operatorname{dim} f^{-1}(f(z)) \geqq n-k$. Thus $k=p$ and $\operatorname{dim}$ $R_{p-1}(f) \leqq n-1$. At each point $z$ of $M^{n}-R_{p-1}(f)$ the map $f$ has the desired form (with $q(z)=1$ ) so that it suffices to consider only points $z$ of $R_{p-1}(f)$, and we may suppose that $\operatorname{dim} R_{p-1}(f)=n-1$.

Let $\Lambda$ be a component of the set of those simple points of $R_{p-1}(f)$ having dimension $n-1$, and apply (3.7) to $f \mid \Lambda: \Lambda \rightarrow N^{p}$. It follows as above that, except for an analytic subset (of $\Lambda$ ) of dimension at most $n-2, f \mid \Lambda$ has rank at least $p-1$.

Let $W_{1}$ and $E_{1}$ be the $C$-analytic sets given by (3.3) for $B=R_{p-1}(f)$, (i.e., $\left.W_{1}=R_{p-1}(f)\right)$, and let $A_{1}=E_{1}$. The conclusion follows from (3.9) applied to $\Gamma=W_{1}-A_{1}$ with $A=A_{2}$.

4.5. Remark. Theorem (4.4) is false for $C^{\infty}$ maps and $A_{1} \cup A_{2} \subset M^{n}$ a closed subset with $\operatorname{dim}\left(A_{1} \cup A_{2}\right) \leqq n-2$, even in case $n=p=1$. Let $X \subset R$ be the Cantor set, and define $f: R \rightarrow R$ by $f(X)=\{0\}, f(x)=x$ for $x \leqq 0, f(x)=$ $x-1$ for $x \geqq 1$, and $f(x)=\left(x-\left(r+2^{-1}\right) \cdot 3^{-k}\right)^{2}-\left(2^{-1} \cdot 3^{-k}\right)^{2}$ for $x$ in an interval $\left(r \cdot 3^{-k},(r+1) \cdot 3^{-k}\right)$ removed at the $k$-th stage in defining $X$. Then $f$ is continuous on $R$ and $C^{\infty}$ on $R-X$, and by [4; p. 189, (2.4)] we may suppose that $f$ is $C^{\infty}$. Since $D f$ changes sign at each point of $X, X \subset B_{f}$, so that $f$ does not satisfy the conclusion of (4.4).

4.6. Corollary. If $f: M^{n} \rightarrow N^{p}$ is $C^{\omega}$ open, then $\operatorname{dim} B_{f} \leqq n-2$.

Proof. Apply (4.2) and (4.4). The $C^{\infty}$ analog is false even for $n=p=2$ [6; p. 97-98, (3.6) and following Remark; cf. also p. 99].

4.7. Examples. The following examples $f: R^{n} \rightarrow R^{p}$ show that (4.6) is sharp for $p>0 ; \Re(z)$ (resp., $\Im(z)$ ) is the real (resp., imaginary) part of the complex number $z$, and $i=\sqrt{-1}$ : (a) $f_{1}(x)=\Re\left(\left(x_{1}+i x_{2}\right)^{d}\right), f_{2}(x)=\Im\left(\left(x_{1}+\right.\right.$ $\left.\left.i x_{2}\right)^{d}\right)$, and $f_{j}(x)=x_{i}(d=2,3, \cdots ; j=2,3, \cdots, p ; n \geqq p \geqq 2)$; (b) $f_{1}(x)=$ $\Re\left(\left(x_{1}+i x_{2}\right)\right)^{d}$ and $f_{i}(x)=x_{i+1}(d=2,3, \cdots ; j=1,2, \cdots, p ; n>p \geqq 1)$.

5. The branch set of a real analytic map. An analytic triangulation [14; p. 1021] of a set $S \subset M^{n}$ is a homeomorphism $\tau: K \rightarrow S$ (where $K$ is a simplicial complex) such that $\tau$ restricted to the interior of any simplex of $K$ is analytic and $\tau^{-1}$ restricted to the image of the interior of any simplex of $K$ is analytic.

5.1. Theorem (Graver). If $V_{i}(i=1,2, \cdots, k)$ are real analytic subsets 
of the real analytic manifold $M^{n}$, then there is an analytic triangulation of $M^{n}$ such that each $V_{i}$ is a subpolyhedron.

For $M^{n}$ an open subset of $R^{n}$ the theorem results from [14; p. 1030, Global Decomposition Theorem and p. 1029, Corollary to the Triangulation Theorem]. In general, there is a $C^{\omega}$ embedding $\lambda: M^{n} \rightarrow R^{p}$ for some $p$ [13], and we apply the special case of the theorem to $R^{p}$ and its analytic subsets $\lambda\left(M^{n}\right)$ and $\lambda\left(V_{i}\right)$ $(i=1,2, \cdots, k)$ to obtain the general result. Theorem (5.1) is also given in [12] and [18; p. 463].

Note that an analytic triangulation in this sense of a real analytic manifold is not necessarily an analytic triangulation in the usual sense [20; p. 77]. This weaker definition is essential both for (5.1) (let $V_{1} \subset R^{2}$ be the set of zeros of $\left.y^{2}-x^{4}\right)$ and for (5.2) (let $f: R^{2} \rightarrow R^{2}$ be defined by $f(x, y)=\left(x,\left(y^{2}-x^{4}\right)^{2}\right)$ ).

5.2. Theorem. Let $f: M^{n} \rightarrow N^{n}$ be $C^{\omega}$. Then there is a real analytic triangulation of $M^{n}$ such that $B_{f}$ is a subpolyhedron.

Proof. There is (5.1) an analytic triangulation of $M^{n}$ such that $R_{n-1}(f)$ and the $C$-analytic sets $W_{i}$ and $E_{i}(i=1,2, \cdots)$ given by (3.3) for $B_{f}$ are subpolyhedra. Suppose that $B_{f}$ is not a subpolyhedron. Then there is a maximal integer $p$ and an open $p$-simplex $\Delta$ with $\Delta \cap B_{f} \neq \varnothing$, while $\Delta \nsubseteq B_{f}$.

Let $S$ be the open star neighborhood of $\Delta$ in $M^{n}$, i.e., the union of all open simplices of $M^{n}$ having $\Delta$ as a face. Suppose that there is a simplex $\sigma \subset S$, $\sigma \neq \Delta$, such that $\sigma \cap B_{f} \neq \varnothing$. By the maximality of $p, \sigma \subset B_{f}$. Since $B_{f}$ is closed, $\Delta \subset B_{f}$, yielding a contradiction. Thus $S \cap B_{f}=\Delta \cap B_{f}$.

Choose the largest $i$ such that $\Delta$ meets $W_{i}$. Thus $\Delta \subset W_{i}$. If $\Delta \cap E_{i} \neq \varnothing$, then $\Delta \subset E_{i}$ also, and since $\Delta \cap B_{f} \neq \varnothing, \Delta \cap W_{i+1} \neq \varnothing$, yielding a contradiction. Thus each point of $\Delta$ is a simple point of $W_{i}$ having dimension $\operatorname{dim} W_{i}$ (3.3).

Now $\Delta$ is a $C$-irreducible $C$-analytic subset of $S$ (see (2.6)). Let $W_{i, v}(i=$ $1,2, \cdots)$ be those $C$-irreducible components of $W_{i}$ having $\operatorname{dim} W_{i, \nu}=\operatorname{dim} W_{i}$. Since $\Delta$ meets each $W_{i, \nu} \cap S$ either in $\Delta$ or in a $C$-analytic subset of dimension less than $p$ [33; p. 155, Corollaire], $\Delta \subset W_{i, \nu}$ for some $\nu$; thus (3.3) $f \mid \Delta$ has constant rank.

Since $\Delta \subset B_{f}$, it follows from the Rank Theorem [10; p. 273] that this rank is $p$. Choose $y \varepsilon \Delta \cap b d y\left(B_{f} \cap \Delta\right)$, and choose an open neighborhood $U$ of $y$ in $S$ such that $U \cap \Delta$ is a $p$-cell and $f \mid(U \cap \Delta)$ is an embedding. If $p \leqq n-2$, it follows from (3.5) that $p=n-2$ and $U \cap \Delta \subset B_{f}$, contradicting the choice of $U$. Since $B_{f} \neq M^{n}, \operatorname{dim} R_{n-1}(f) \leqq n-1(3.7)$, so that $\operatorname{dim} W_{1} \leqq n-1$; thus $p=n-1$ and $i=1$. By (3.9) (or (3.10)) $\Delta \subset B_{f}$, contradicting its choice. Thus $B_{f}$ is a subpolyhedron of $M^{n}$.

For $C^{\infty}$ maps the theorem is false [6; p. 97, (3.5)]. See also (5.5).

5.3. Remark. If $M^{n}$ is a $C^{\omega}$ manifold, then any compact $C$-analytic subset $W \subset M^{n}$ is a finite polyhedron (5.1) so that the Borel-Moore homology $H_{i}(W$; $Z_{2}$ ) agrees with simplicial homology $[3 ;$ p. 148, Sect. 5]. If $\operatorname{dim} W=w$, then 
$H_{w}\left(W ; Z_{2}\right)$ has in a natural sense a fundamental class in dimension $w$ [2] so that $H_{w}\left(W ; Z_{2}\right) \neq 0$. Let $X$ be a $C$-analytic subset of $W$ with $\operatorname{dim} X<w$. Then (5.1) $H_{w}\left(C l[W-X] ; Z_{2}\right) \neq 0$ also.

5.4. Theorem. Let $f: M^{n} \rightarrow N^{n}$ be $C^{\omega}$ and let $\operatorname{dim} B_{f}=q$. Then

(a) there is a $C$-analytic subset $V \subset M^{n}$ such that $\operatorname{dim} V=q$ and $B_{f} \subset V$.

(b) There is a C-analytic subset $E \subset M^{n}$ such that $\operatorname{dim} E<q$, and, if $q \neq$ $n-2, V-E \subset B_{f}$.

(c) If $n \leqq 2$, then $B_{f} \subset M^{n}$ is a $C$-analytic subset.

(d) If $M^{n}$ is compact, $q \neq n-2$, and $B_{f} \neq \varnothing$, then $H_{\alpha}\left(B_{f} ; Z_{2}\right) \neq 0$.

(e) If $M^{n}$ is compact and $n>1$, then either $H_{j}\left(B_{f} ; Z_{2}\right) \neq 0$ for some $j>0$ or $f \mid\left(M^{n}-E\right)$ is open.

The set $B_{f}$ is a polyhedron (5.2), and the homology is simplicial homology.

Proof. We may suppose that $B_{f}$ is not a component of $M^{n}$, so that the $C$-analytic set $R_{n-1}(f)$ has dimension at most $n-1$ (3.7). Let $V$ be the smallest $C$-analytic subset of $M^{n}$ containing $B_{f}$ (3.2). By the Inverse Function Theorem, $B_{f} \subset R_{n-1}(f)$ so that $V \subset R_{n-1}(f)$ and $\operatorname{dim} V \leqq n-1$.

Let $\left\{V_{\nu}\right\}(\nu=1,2, \cdots)$ be the (locally finite) family of $C$-irreducible components of $V$, and let $\rho(\nu)=\operatorname{dim}\left(V_{\nu}\right)$. Then $V_{\nu}^{*}$ are the irreducible components of $V^{*}$ [33; p. 155, Proposition 11].

Let $E_{\nu} \subset V_{\nu}$ be the $C$-analytic subset given by (3.1) such that $\operatorname{dim} E_{\nu} \leqq$ $\rho(\nu)-1$, each point of $V_{\nu}-E_{\nu}$ is a simple point of dimension $\rho(\nu)$ and $f \mid\left(V_{\nu}-E_{\nu}\right)$ has constant rank $r(\nu)$, and let $F_{\nu}=E_{\nu} \cup \bigcup_{\mu \neq \nu}\left(V_{\mu} \cap V_{\nu}\right)$; then $\operatorname{dim} F_{\nu}<\rho(\nu)$ [33; pp. 155-156, Corollaire]. If $r(\nu)<\rho(\nu)$, let $G_{\nu}=F_{\nu}$; it follows then from the Rank Theorem [10; p. 273] that $V_{\nu}-G_{\nu} \subset B_{f}$.

Suppose that $r(\nu)=\rho(\nu)$. For each $x \varepsilon V_{\nu}-F_{\nu}$ there is an open neighborhood $U$ of $x$ in $M^{n}$ such that $U \cap V=U \cap\left(V_{\nu}-F_{v}\right), U \cap\left(V_{\nu}-F_{v}\right)$ and $f(U \cap$ $\left.\left(V_{\nu}-F_{\nu}\right)\right)$ are $\rho(\nu)$-cells, and $f \mid U$ is light (2.3). By the minimality of $V, B_{f} \cap$ $\left(V_{\nu}-F_{\nu}\right) \neq \varnothing$ so that (3.5) $\rho(\nu)=n-1$ or $n-2$. If $\rho(\nu)=n-2$, let $G_{\nu}=$ $F_{\nu}$; then $\left(\mathfrak{P}_{\nu}\right)$ for every $z \varepsilon V_{\nu}-G_{\nu},{ }_{z} f$ is $C^{0}$ equivalent to ${ }_{0} F_{n, d(z)}(d(z)=$ $1,2, \cdots)$, and for some $z, d(z)>1$.

Finally, suppose that $r(\nu)=\rho(\nu)=n-1$. Let $S_{\nu}$ be the intersection of $V$, with the singular set $S_{\nu}^{\prime}$ of $R_{n-1}\left(f^{*}\right)$, and let $G_{\nu}=F_{v} \cup S_{\nu}$; then [33; p. 155, Proposition 12] $\operatorname{dim} G_{\nu} \leqq n-2$. Since $V_{\nu}^{*}$ is irreducible, $V_{\nu}^{*}-\left(F_{\nu}^{*} \cup S_{\nu}^{\prime}\right)$ is a connected complex analytic $(n-1)$-manifold [21; p. 68, Corollary 2 ], so that the natural number $k$ given by (3.9) for $f^{*}$ at each point $y$ of $V_{\nu}^{*}-\left(F_{\nu}^{*} \cup S_{\nu}^{\prime}\right)$ is independent of $y$. For $y \varepsilon V_{\nu}-G_{\nu}$ this is the same number given by (3.9) for $f$. By the minimality of $V, B_{f}$ meets $V_{\nu}-G_{\nu}$, so that $k$ is even; as a result, $V_{\nu}-G_{\nu} \subset B_{f}$.

In summary, for each $\nu$ either $V_{\nu}-G_{\nu} \subset B_{f}$ or $\mathfrak{B}_{\nu}$ is true. Conclusion (a) follows. If $q=n$, let $E=\varnothing$; otherwise, let $E$ be the union of the $V_{\nu}$ for $\rho(\nu)<q$ and $G_{\nu}$ for $\rho(\nu)=q\left[33 ;\right.$ p. 154]. Then (b) $V-E \subset B_{f}$, unless $q=n-2$ and some $\mathfrak{B}_{\nu}$ is true. 
In particular, if $n=2$ and $B_{f} \neq M^{n}$, then $\operatorname{dim} V \leqq 1$. Thus $V-B_{f} \subset E$ where $\operatorname{dim} E \leqq 0$, i.e., $E$ is a discrete set (5.1), and we may suppose that $E \cap$ $B_{f}=\varnothing$. There is an open neighborhood $\Psi$ of $M$ in $M^{*}$ small enough that the components of $V^{*} \cap \Psi$ meeting $B_{f}$ do not meet $E$. The union $B^{*}$ of these components is a complex analytic subset of $\Psi$ with $B^{*} \cap M=B_{f}$, so that $B_{f}$ is $C$-analytic (i.e., $B_{f}=V$ ).

If $M^{n}$ is compact, then (5.3) $H_{\rho(\nu)}\left(V_{\nu} ; Z_{2}\right) \approx H_{\rho(\nu)}\left(C l\left[V_{\nu}-G_{\nu}\right] ; Z_{2}\right) \neq 0$. Suppose, in addition, that $\mathfrak{B}_{\nu}$ is false. Since $B_{f} \subset V, F_{\nu} \subset G_{\nu}$, and $V_{\nu}-G_{\nu} \subset$ $B_{f}$, we have $H_{\rho(\nu)}\left(B_{f} ; Z_{2}\right) \neq 0$ also. Conclusion (d) follows. Moreover, $H_{j}\left(B_{f}\right.$; $\left.Z_{2}\right) \neq 0$ for some $j>0$ unless for every $\nu$ either $\rho(\nu)=0$ or $\mathfrak{B}_{\nu}$ is true; i.e., by the minimality of $V$, either $\operatorname{dim} B_{f}=0$ or for every $\nu, \Re_{\nu}$ is true. Conclusion (e) follows from $[6 ;$ p. 94, (2.3)].

5.5. Remark. We do not know (for $n \geqq 3$ ) whether (*) $B_{f}$ is a $C$-analytic (or analytic) set. If $(*)$ were proved, then (5.2) and (5.4) would be corollaries; otherwise, they may be sharp.

It appears worthwhile to indicate the precise difficulties in extending the proof of (5.4) to a proof of $(*)$, since (1) probably a proof of $(*)$ would involve in large part the proof of (5.4), and (2) the difficulties indicate something of the nature of possible counter-examples.

If for some $\nu(I) \operatorname{dim} V_{\nu}=n-2$ and $f \mid\left(V_{\nu}-G_{\nu}\right)$ is an embedding, then $\left(\mathfrak{B}_{\nu}\right)$ for every $z \varepsilon V_{\nu}-E_{\nu}, f$ is $C^{0}$ equivalent to $F_{n, d(z)}(d(z)=1,2, \cdots)$ and some $d(z)>1$. While $d$ is constant on each component of $V_{\nu}-E_{\nu}$, it is not clear that it is constant. To prove (*) (or even (5.4b)), one must prove that $d(z)>1$ for every $z \varepsilon V_{\nu}-E_{\nu}$. (In case $\operatorname{dim} V_{\nu}=n-1$ and $f \mid\left(V_{\nu}-E_{\nu}\right)$ is an embedding, a complexification argument gives the analogous conclusion.)

For a $C$-analytic set $V$ let $A(V)$ be the set of those simple points $y \varepsilon V$ with $\operatorname{dim}_{y} V=\operatorname{dim} V$. The proof of (5.4) shows that $\bar{A}\left(V_{v}\right) \subset B_{f}$ except in case $(I)$. There are, however, $C$-irreducible $C$-analytic subsets $V \subset R^{n}$ with $\bar{A}(V) \neq V$ $[21 ;$ p. 106 , Example 1], unlike the corresponding complex situation $[21 ;$ p. 66 , Proposition 2, and p. 68, Corollary 4]. Thus, it is not clear that $V_{\nu} \subset B_{f}$.

Complexification, which is used in (5.4), seems a natural approach, but it is difficult to relate topological properties of $f$ to topological (and analytic) properties of $f^{*}$.

5.6. Examples. For $C^{\infty}$ maps (5.4) (d) and (e) are false. Let $S^{n}$ be the $n$ sphere and let $B$ be any compact connected set with $S^{n}-B$ homeomorphic to $R^{n}$, e.g., a (tame) $k$-cell $(k=1,2, \cdots, n)$ or the graph of the $\sin 1 / x$ curve. Assume $n \neq 4$ so that $S^{n}-B$ is $C^{\infty}$ diffeomorphic to $R^{n}$ [28; p. 481 and p. 487, (5.2)]. Let $y \varepsilon S^{n}$ and let $f: S^{n} \rightarrow S^{n}$ be defined by $f(B)=\{y\}$ and $f$ maps $S^{n}-B$ $C^{\infty}$ diffeomorphically onto $S^{n}-\{y\}$. We may suppose that $f$ is $C^{\infty}[4 ;$ p. 189, (2.4)].

In one topological situation, however, $B_{f}$ is necessarily cyclic.

5.7. Remark. Let $M^{n}$ and $N^{n}$ be connected and oriented, and let $f: M^{n} \rightarrow N^{n}$ 
be $C^{1}$ and proper. If the Jacobian determinant changes sign, then, for any module $G(\neq 0)$ over a commutative ring, $H_{c}^{n-1}\left(B_{f} ; G\right) \neq 0$.

We will actually deduce the conclusion under the topological hypothesis: $M^{n}$ and $N^{n}$ are oriented, $f: M^{n} \rightarrow N^{n}$ is $\left(C^{0}\right.$ and) proper, and there exist $x$ and $y$ in $M^{n}-B_{f}$ at which the local degree (of $f$ ) $d_{x}=1$ and $d_{y}=-1$. For relevant terminology and information see [30] and [29; pp. 115, 116, and 127].

Proof. Define $X_{i} \subset M^{n}-B_{f}(i=1,2)$ by: $x \varepsilon X_{1}$ (resp., $X_{2}$ ) if and only if there is a neighborhood $U$ of $x$ such that $d_{y}>0$ (resp., $d_{y}<0$ ) for every $y \varepsilon U-$ $B_{f}$. Then $M^{n}-B_{f}=X_{1} \cup X_{2}$. Let $Y_{i}=B_{f} \cup X_{i}$; then each $Y_{i}$ is closed in $M^{n}, Y_{1} \cap Y_{2} \subset B_{f}$, and $Y_{1} \cup Y_{2}=M^{n}$. From the Mayer-Vietoris sequence, $H_{c}^{n-1}\left(Y_{1} \cap Y_{2} ; G\right) \neq 0$, and, since $H_{c}^{n}\left(B_{f}, Y_{1} \cap Y_{2} ; G\right)=0$, the desired conclusion follows from the cohomology sequence (see [27; p. 320 and p. 322, (12)]).

5.8. Corollary. Let $M^{n}$ and $N^{n}$ be connected and oriented $C^{1}$ manifolds, and let $f: M^{n} \rightarrow N^{n}$ be a $C^{1}$ proper map of degree 0 . Then, for any module $G(\neq 0)$ over a commutative ring, either $H_{c}^{n}\left(B_{f} ; G\right) \neq 0$ or $H_{c}^{n-1}\left(B_{f} ; G\right) \neq 0$.

Proof. If the Jacobian determinant $J \equiv 0$, then $f\left(M^{n}\right)=f\left(R_{n-1}(f)\right)$ has dimension at most $n-1[29 ;$ p. $47,(3.1)]$, so that $B_{f}=M^{n}$. Suppose the conclusion is false; then $J \not \equiv 0$ and (5.7) $J \geqq 0$ (say). Choose an open neighborhood $U$ with $f \mid U$ a diffeomorphism; since $\operatorname{dim}\left(f\left(R_{n-1}(f)\right)\right) \leqq n-1[6 ;$ p. 88, (1.3)], there is a regular value $y \varepsilon f(U)-f\left(R_{n-1}(f)\right)$. It follows from [29; p. 127, (4.2)] that the degree of $f$ is positive, contradicting the hypothesis.

Corollary (5.8) generalizes [7; p. 386, (3.8)].

\section{Some questions and related information.}

6.1. Remark. $A C^{\omega}$ proper acyclic $\bmod 2 \operatorname{map} f: M^{n} \rightarrow N^{p}$ with $n \geqq p$ is a finite-to-one covering map.

Proof. For each $y \varepsilon N^{p}$ either $f^{-1}(y)=\varnothing$ or $H_{j}\left(f^{-1}(y) ; Z_{2}\right) \neq 0$ where $j=$ $\operatorname{dim} f^{-1}(y)$ (5.3); thus, $f^{-1}(y)$ has at most one point. From (3.7) $n=p$ and from (4.4) $\operatorname{dim} B_{f} \leqq n-2$, so that [6; p. 94, (2.3)] $f$ is open. Thus [6; p. 91, (2.1); it extends to proper maps] $f$ is a local homeomorphism, and so [23; p. 128] is a $k$-to-one covering map for some natural number $k$.

6.2. Proposition. Let $f: M^{n} \rightarrow N^{n}$ be $C^{\omega}$ and proper with $n \geqq 4$ and $\operatorname{dim}$ $B_{f}<n / 2$. Then $f$ is a finite-to-one covering map.

Proof. A proper map $g: M^{n} \rightarrow K^{n}$ is called homotopy cellular if, for each $y \varepsilon K^{n}$, there are compact contractible $n$-manifolds $A_{i}(j=1,2, \cdots)$ with simply connected boundary such that $A_{i+1} \subset \operatorname{int}\left(A_{i}\right)$ and $g^{-1}(y)=\bigcap_{i} A_{i}$. Each $A_{j}$ has the Alexander-Spanier mod 2 cohomology of a point [27; p. 398, (5), p. 243 , (3), and p. $340,(5)]$, so that $[27$; p. $318,(6)$ and p. $334,(8)] g^{-1}(y)$ has the Cech mod 2 cohomology of a point, i.e., $g$ is acyclic mod 2 (2.3). Thus (6.1) $g$ is a homeomorphism. 
By [4; p. 186, (1.2)], $f=h \circ g$, where $g$ is $C^{\omega}$ homotopy cellular and $h$ is a $k$-to-1 $C^{\omega}$ diffeo-covering map for some natural number $k$, and the conclusion follows.

6.3. Theorem. [7; p. 370]. Let $f: M^{n} \rightarrow N^{n}$ be $C^{m}(m=3,4, \cdots ; m=\infty$; or $m=\omega)$ and proper with $\operatorname{dim} B_{f} \leqq n-3$. Then there is a factorization $f=$ $h \circ g$ such that

(1) $g: M^{n} \rightarrow K^{n}$ is a $C^{m}$ monotone map onto the $C^{m}$ manifold $K^{n}$; and

(2) $h: K^{n} \rightarrow N^{n}$ is a k-to-1 $C^{m}$ diffeo-covering map. Moreover,

(3) if $\bar{h} \circ \bar{g}$ is another such factorization with intermediate space $L^{n}$, then there is a $C^{m}$ diffeomorphism $\alpha$ of $K^{n}$ onto $L^{n}$ such that $\bar{g}=\alpha \circ g$ and $\bar{h}=h \circ \alpha^{-1}$.

6.4. Questions. The above results suggest the following questions:

(1) If $f$ in (6.3) is $C^{\omega}$, is it a finite-to-one covering map? This would extend (6.2).

(2) More generally, is a $C^{\omega}$ proper monotone onto map $g: K^{n} \rightarrow N^{n}$ a homeomorphism?

(3) In particular, let $g: S^{p} \times S^{\alpha} \rightarrow S^{p+a}$ be the $\left(C^{\infty}\right)$ smash product map [4; p. 189, (2.5)]; one point-inverse is the one-point union $S^{p} \vee S^{\alpha}$, and the others consist of single points. Is it true that no $C^{\omega}$ map is $C^{0}$ equivalent (2.2) to $g$ ? (Compare with (6.2) and (6.5).)

(4) If the map $f$ of (6.3) is $C^{\omega}$ but not necessarily proper, does there exist such a factorization where $h$ is (merely) a $C^{\omega}$ local homeomorphism? (See (6.6).)

(5) If $M^{n}$ and $N^{n}$ are connected and oriented, $N^{n}$ is simply connected, $n \geqq 2$, $f: M^{n} \rightarrow N^{n}$ is a $C^{m}(m=0,1, \cdots ; m=\infty$; or $m=\omega)$ proper map with deg $f \neq \pm 1$, is $H^{i}\left(B_{f} ; Z_{2}\right) \neq 0$ for $j=n-2, n-1$, or $n$ ? (See (6.3), [7; p. 370, (1.2)], (5.4), (5.7) and (5.8). In case $m=\omega$, if $B_{f}$ is in general an analytic subset of $M^{n}(5.5)$, then $H_{q}\left(B_{f} ; Z_{2}\right) \neq 0$ where $q=\operatorname{dim} B_{f}(5.3)$.)

6.5. Examples. Without the properness hypothesis (6.1), (6.2) and (6.4) (1) and (2) are false. Define $f: F^{n} \rightarrow F^{n}$ by $f_{i}(x)=x_{i}(i<n)$ and $f_{n}(x)=\left(\sum_{i<n}\right.$ $\left.\left(x_{i}\right)^{2}\right) \cdot x_{n}(F=R$ or $C)$.

For $C^{\infty}$ proper maps the same statements are false ((5.6) and [4; $(2.5),(2.12)$, (2.14), (2.15), and (4.5)]).

6.6. Remark. In the case of $C^{\infty}$ maps, (6.4) (4) has an affirmative answer if and only if $n \leqq 3$.

Proof. For $n \leqq 3$ see $[7 ;$ p. 385, (3.6)].

For $n \geqq 4$, however, the examples of $[6 ;$ p. 98, Remark after (3.6)] with

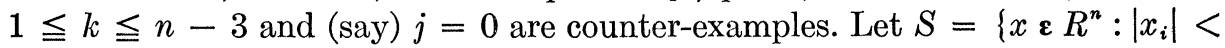
$1(i=1,2, \cdots, n)\}$, and let $g^{\prime}: S \rightarrow g^{\prime}(S) \subset R^{n}$ be defined by $\left(g^{\prime}\right)_{r}(x)=x_{r}$ $(r \leqq n-k)$ and $\left(g^{\prime}\right)_{r}(x)=x_{r} \cdot\left[\sum_{i \leqq n-k}\left(x_{i}\right)^{2}\right]^{1 / 2}(n-k<r)$; then $g^{\prime}$ is the analog of the map $h$ (not of $g$ ) in $[6 ;(3.6)]$. Let $h^{\prime}: g^{\prime}(S) \rightarrow R^{n}$ be the analog of $g$ in $[6 ;(3.6)]$, a light map (2.3) which fails to be locally one-to-one at 0 . Define $f=h^{\prime} \circ g^{\prime}$. 
Suppose that $f$ has a factorization $f=h \circ g$ of the desired form, even with $g$ not necessarily onto. Then $g: S \rightarrow g(S)$ and $h \mid g(S)$ define a monotone-light factorization [34; p. 141] of $f$, as also do $g^{\prime}$ and $h^{\prime}$, and from the (topological) uniqueness of such factorizations (properness is not necessary), we may suppose that $g^{\prime}(S) \subset K^{n}$ and $h \mid g^{\prime}(S)=h^{\prime}$. Since $h^{\prime}$ is not locally one-to-one at 0 , while $h$ is, a contradiction results.

\section{REFERENCES}

[1] S. Bochner \& W. T. Martin, Several Complex Variables, Princeton University Press, Princeton, New Jersey, 1948.

[2] A. Borel \& A. Haffriger, La classe d'homologie fondamentale d'un espace analytique, Bull. Soc. Math. France, 89 (1961) 461-513.

[3] A. Borex \& J. C. Moore, Homology theory for locally compact spaces, Michigan Math. $J ., 7$ (1960) 137-159.

[4] P. T. Church, Differentiable monotone maps on manifolds, Trans. Amer. Math. Soc., 128 (1967) 185-205.

[5] — Differentiable open maps, Bull. Amer. Math. Soc., 68 (1962) 468-469.

[6] - Differentiable open maps on manifolds, Trans. Amer. Math. Soc., 109 (1963) $87-100$.

[7] - Factorization of differentiable maps with branch set dimension at most $n-3$, Trans. Amer. Math. Soc., 115 (1965) 370-387.

[8] - On points of Jacobian rank k, Trans. Amer. Math. Soc., 110 (1964) 413-423.

[9] — E. Hemmingsen, Light open maps on $n$-manifolds, II, Duke Math. J., 28 (1961) 607-624.

[10] J. Dieudonne, Foundations of Modern Analysis, Academic Press, New York and London, 1960.

[11] S. Eilenberg \& N. Stemnrod, Foundations of Algebraic Topology, Princeton University Press, Princeton, New Jersey, 1952.

[12] B. Gresecke, Simpliziale Zerlegung abzählbarer analytischer Räume, Math. Z., 83 (1964) 177-213.

[13] H. Grauert, On Levi's problem and the imbedding of real analytic manifolds, Ann. of Math., 68 (1958) 460-472.

[14] J. E. Graver, An analytic triangulation of an arbitrary real analytic variety, J. Math. Mech., 13 (1964) 1021-1036.

[15] R. C. Gunning \& H. Rossi, Analytic Functions of Several Complex Variables, PrenticeHall, Englewood Cliffs, New Jersey, 1965.

[16] M. Henve, Several Complex Variables, Local Theory, Oxford University Press and Tata Institute of Fundamental Research, London, 1963.

[17] W. Hurewicz \& H. Wallman, Dimension Theory, Princeton University Press, Princeton, New Jersey, 1948.

[18] S. Lojasiewicz, Triangulation of semi-analytic sets, Ann. Scuola Norm. Sup. Pisa, 18 (1964) 449-474.

[19] J. Milnor, Differential Topology, Princeton University mimeographed notes, 1958.

[20] J. R. Munkres, Elementary Differential Topology, Princeton University Press, Princeton, New Jersey, 1963.

[21] R. Narasimhan, Introduction to the Theory of Analytic Spaces, Springer Verlag, Lecture Notes in Mathematics, No. 25, Berlin, 1966.

[22] W. D. Nathan, Open Mappings on Manifolds, Doctoral Dissertation, Syracuse University, 1968.

[23] R. S. Palais, Natural operations on differential forms, Trans. Amer. Math. Soc., 92 (1959) $125-141$. 
[24] R. Remmert, Holomorphe und meromorphe Abbildunger komplexer Räume, Math. Ann., 133 (1957) 328-370.

[25] R. Remmert \& K. Stein, Eigentliche holomorphe Abbildungen, Math. Z., 73 (1960) 159189.

[26] H. B. Shutrick, Complex extension, Quart. J. Math. Oxford Ser., 9 (1958) 189-201.

[27] E. H. Spanier, Algebraic Topology, McGraw-Hill, New York, 1966.

[28] J. Stallings, The piecewise linear structure of Euclidean space, Proc. Cambridge Philos. Soc., 58 (1962) 481-488.

[29] S. Stmrnberg, Lectures on Differential Geometry, Prentice-Hall, Englewood Cliffs, New Jersey, 1964.

[30] $\&$ R. G. Swan, On maps with nonnegative Jacobian, Michigan Math. J., 6 (1959) 339-342.

[31] R. Tном, Quelques propriétés globales des variétés différentiables, Comment. Math. Helv., 28 (1954) 17-86.

[32] H. Whitney, Tangents to an analytic variety, Ann. of Math., 81 (1965) 496-549.

[33] — F. Bruhat, Quelques propriétés fondamentales des ensembles analytiquesréels, Comment. Math. Helv., 33 (1959) 132-160.

[34] G. T. Whyburn, Analytic Topology, Amer. Math. Soc. Colloq. Publ. vol. XXVIII, Amer. Math. Soc., New York, 1942.

Institute for Advanced Study,

Syracuse University, and

Temple University

Date communicated: OCTOBER 23, 1968 